\title{
Flightless I Homolog Reverses Neo-Endocrine Therapy Resistance through YBX1/PD-L1 Axis Mediated-Immune Evasion in Prostate Cancer
}

\section{Hailong Ruan}

wuhan union hospital

Lin Bao

Wuhan Union Hospital

Zhen Tao

Tianjin Medical University

Ke Chen ( $\nabla$ shenke@hust.edu.cn )

Huazhong University of Science and Technology

\section{Research}

Keywords: FLII, YBX1, PD-L1, enzalutamide-resistance, immune evasion

Posted Date: August 1st, 2020

DOI: https://doi.org/10.21203/rs.3.rs-48777/v1

License: (c) (i) This work is licensed under a Creative Commons Attribution 4.0 International License. Read Full License 


\section{Abstract}

Background: Tumor cells can evade immune surveillance and immune killing effects during neoendocrine therapy resistance, but the mechanisms that induce this phenomenon are still unclear. Flightless I homolog (FLII) functions as a coregulator of transcription factors in malignancies. Here, we aim to identify the functions and mechanisms of FLII-mediated neo-endocrine therapy resistance and immune evasion in prostate cancer (PCa).

Methods: Bioinformatics analysis and PCa tissue microarray were used to assess the correlation between FLII and the immune checkpoint PD-L1. Immunoprecipitation (IP) and chromatin immunoprecipitation (ChIP) were used to investigate the regulation of PD-L1 expression by FLII. Enzyme-linked immunosorbent assay (ELISA) was used to study the effect of FLII on cytokine secretion. The number of effector T cells and immunosuppressive lymphocytes was detected by flow cytometry (FCM).

Results: FLII was negatively correlated with the expression of PD-L1 in PCa. Mechanistic studies demonstrated that FLII physically interacted with YBX1 to inhibit the nuclear localization of YBX1 and thereby suppressed the transcriptional expression of PD-L1 in enzalutamide-resistant tumors. Restoration of FLII expression reversed neo-endocrine therapy resistance by activating the $T$ cell response in the tumor microenvironment mediated by partial inhibition of the YBX1/PD-L1 pathway. The reversal of neoendocrine therapy resistance and immune evasion was mediated by the proliferation of effector CD8+ $T$ cells and inhibition of the infiltration of regulatory $T$ cells and myeloid-derived suppressor cells in the tumor microenvironment.

Conclusions: FLII inhibits tumor progression in part through repressing YBX1/PD-L1-mediated immune evasion. Combination therapy with FLII and neo-endocrine therapy may improve the benefits in prostate cancer patients and avoid immune evasion.

\section{Background}

Prostate cancer ( $\mathrm{PCa}$ ) was the most frequent malignancy and the second major cause of cancer-related death among males in the US in 2018 [1], while prostate cancer was the second most frequent malignancy in men worldwide [2]. Androgen receptor (AR) plays a crucial role in normal prostate development and homeostasis and drives prostate tumorigenesis and tumor progression [3]. Therefore, neo-endocrine therapy-based androgen deprivation therapy (ADT) is the main treatment mode for prostate cancer. Prostate cancer patients are initially sensitive to ADT but slowly become hormoneinsensitive or even resistant over time, resulting in poor prognosis [4]. Prostate cancer naturally progresses from hormone-sensitive to hormone-resistant and eventually inevitably develops into castration-resistant prostate cancer (CRPC). Currently, neo-hormonal therapies for CRPC include abiraterone and enzalutamide (ENZ). However, the optimal treatment regimen for CRPC patients remains unclear and requires extensive further research and clinical trials. 
In the past few years, tumor immunotherapy has become an emerging form of cancer treatment. Although prostate cancer is a recognized immunogenic tumor [5], it can evade immune responses by producing immunosuppressive cytokines such as TGF- $\beta$, inducing T cell apoptosis, or increasing the number of regulatory T cells (Tregs) [6]. Moreover, prostate cancer is considered a "cold" cancer with minimal $T$ cell infiltration and minimal response to single immune checkpoint inhibitors, and this has been confirmed by multiple studies [7-10]. The lack of responses to immune checkpoint inhibitors in these studies may be due to the extremely low expression of PD-L1 in prostate cancer [11]. Based on the above statement, any single immunotherapy is unlikely to significantly alter the outcome of prostate cancer treatment, but combination therapy strategies show a more promising and optimistic situation [12]. Several large studies have shown that the combination of immune checkpoint inhibitors with radiotherapy, DNA-damaging agents, hormonal therapy, or chemotherapy can enhance immune system responses and induce long-lasting clinical responses without obvious toxic side effects [13-15]. Therefore, the current treatments for CRPC may be more beneficial if they combine new neo-endocrine therapy with immune checkpoint inhibitors.

The flightless I homolog (FLII) gene, first discovered in Drosophila melanogaster, encodes a protein with a C-terminal gelsolin-like actin binding domain (GLD) and an N-terminal leucine-rich repeat (LRR) proteinprotein interaction domain. FLII is a member of the conserved gelsolin family, which contains a variety of proteins from eukaryotes. The functions of these proteins include cleaving, nuclear localization, capping, and bundling of actin filaments [16]. Gelsolin, the core of this protein family, has been reported to be involved in cell mobility, cell apoptosis, signaling pathways and tumor suppression [17]. FLII can interact with a variety of proteins to regulate intracellular signaling in tumors [18-20]. Mammalian FLII has been reported to function as a coactivator via nuclear hormone receptor signaling, including signaling via estrogen receptors and thyroid hormone receptors [21]. We have previously discovered that FLII, as a cosuppressor of AR, can antagonize the DHT-induced nuclear localization of AR, thereby reversing the tolerance of traditional neo-endocrine therapy (bicalutamide) and enhancing the sensitivity to neoendocrine therapy (enzalutamide) [22]. However, the role and mechanism of FLII in enzalutamideresistant CRPC remain unclear.

In this study, we found that PD-L1 expression was upregulated in enzalutamide-resistant cells (C4-2-R cells) compared to parental cells (C4-2 cells). Bioinformatics analysis and tissue microarray (TMA) analysis of prostate cancer found a negative correlation between FLII and PD-L1 protein expression. The drug resistance gene YBX1 could transcriptionally regulate the expression of PD-L1 in human and murine prostate cancer cells. Through binding to the YBX1 protein, FLII antagonized the entry of YBX1 into the cell nucleus, thereby inhibiting the transcription of PD-L1 and enhancing the immune response of prostate cancer. Restoration of FLII expression enhanced the sensitivity of CRPC cells to enzalutamide treatment and activated T cells by blocking the immune checkpoint PD-L1 in vitro and in vivo. Our current study indicates that FLII regulates PD-L1 expression through YBX1 signaling. In summary, FLII inhibits tumor progression in part through repressing YBX1/PD-L1-mediated immune evasion. FLII may serve as a potential therapeutic target for CRPC. 


\section{Methods}

\section{Cell culture, plasmid construction, lentiviral particle infection, transfection, reporter genes and reagents}

The human PCa cell lines C4-2 and PC3 were purchased from ATCC. ENZ-resistant C4-2 (C4-2-R) cells were generated in our lab through continuous ENZ stimulation. The mouse prostate cancer cell line RM-1 was purchased from Shanghai GeneChem Co., Ltd. Cells were maintained in RPMI-1640 medium containing $10 \% \mathrm{FBS}$ and $1 \%$ penicillin/streptomycin and were cultured in a $5 \% \mathrm{CO} 2$ incubator at $37^{\circ} \mathrm{C}$. All cell lines were examined and free of mycoplasma contamination. Small hairpin RNA (sh-RNA) against human or mouse YBX1 (sh-YBX1), FLII (sh-FLII) and the corresponding vector with nonsense sequences were purchased from Shanghai GeneChem. The lentiviral particles containing GV341-FLAG-YBX1 and GV348-HA-FLII were purchased from Shanghai GeneChem and used to infect cells according to the manufacturer's recommendations. The p-FLAG-YBX1-CSD, p-FLAG-YBX1-C-term, p-HA-FLII-LRR, and p-HAFLII-GLD plasmids were kindly provided by Professor Chen [22]. Lipofectamine 2000 was used for plasmid transfection as recommended by the manufacturer. Human and mouse luciferase reporters containing the PD-L1 promoter region were purchased from Shanghai GeneChem Co., Ltd.

\section{PCa tissue samples}

Human PCa tissue microarrays (TMAs) (catalogue: PC1601) were purchased from Wuhan Servicebio Biotechnology Co., Ltd. All information, including pathological diagnosis and clinical stage, was downloaded from the online database (http://www.servicebio.cn/).

\section{PCa data from TCGA and the ONCOMINE database}

Normalized PCa mRNA expression datasets were downloaded from cBioPortal and Oncomine and used to assess the association between FLII and PD-L1 transcripts. All information, including pathological diagnosis and clinical stage, was downloaded from the cBioPortal and Oncomine websites (http://www.cbioportal.org/ and https://www.oncomine.org/).

\section{qRT-PCR assays}

Total cellular RNA was extracted using TRIzol reagent (Life Technologies, CA, USA) according to the manufacturer's recommendations. Specific experimental protocols were performed as previously described [23]. The primer sequences used are as follows:

PD-L1:

Forward, 5'-GTACCTTGGCTTTGCCACAT-3',

Reverse, 5'-CCAACACCACAAGGAGGAGT -3';

FLII: 
Forward, 5'- GCAGGACTGCTACGTCTTCC-3',

Reverse, 5'- TTCTTTTGCAGGCTGAAGGT-3',

AR-V7:

Forward, 5'-CCATCTTGTCGTCTTCGGAAATGTTA-3',

Reverse, 5'-TTTGAATGAGGCAAGTCAGCCTTTCT-3';

GAPDH:

Forward, 5'-GAGTCAACGGATTTGGTCGT-3',

Reverse, 5'-GACAAGCTTCCCGTTCTCAG-3'.

\section{Immunohistochemistry (IHC) and immunofluorescence (IF) assays}

IHC analysis of human PCa tissues was performed using a monoclonal FLII antibody (sc-21716, Santa Cruz), a monoclonal PD-L1 antibody (ab205921, Abcam) and a monoclonal CD8A antibody (ab17147). $\mathrm{IHC}$ results were then evaluated by two independent pathologists with no knowledge of the tissue sample information. The immunoreactive score (IRS) ranges from 0 to 12 , which is a result of multiplication of the staining intensity score ( $0-3)$ and the positive cell proportion score (0-4). The IRS (score $0-1$, negative; score 2-3, mild; score 4-8, moderate; and score 9-12, strong positive) gauged the expression of FLII, PD-L1 and CD8A. IF staining was performed using a monoclonal HA antibody (\#2367, Cell Signaling Technology) and a polyclonal YBX1 antibody (20339-1-AP, Proteintech). Briefly, adherent cells were fixed with $4 \%$ paraformaldehyde, permeabilized with $0.3 \%$ Triton X-100, blocked using $3 \%$ BSA for 1 $\mathrm{h}$ at $37^{\circ} \mathrm{C}$, followed by incubation with primary antibodies and fluorescent secondary antibodies.

\section{Luciferase reporter assays}

A truncated human and mouse PD-L1-promoter luciferase reporter and a control reporter were constructed by Shanghai GeneChem. A Renilla luciferase reporter and the truncated PD-L1 promoter reporter were cotransfected into cells overexpressing FLII and with YBX1 knocked down or cells overexpressing YBX1 using Lipofectamine 2000. Luciferase activity was measured at $48 \mathrm{~h}$ after transfection using Renilla luciferase for normalization.

\section{Western blotting assays and immunoprecipitation (IP)}

Cells were lysed with RIPA buffer containing proteinase inhibitors. Extracted proteins were subjected to SDS-PAGE and then transferred to PVDF membranes. The PVDF membranes were blocked with $5 \%$ milk and incubated with primary antibodies overnight at $4{ }^{\circ} \mathrm{C}$. The next day, the membranes were washed with PBS and incubated with secondary antibodies. IP was performed by adding the primary antibody and magnetic beads to the lysed cell supernatant, and then the centrifuged cell supernatant was subjected to 
SDS-PAGE. The subsequent steps were the same as those in the western blotting experiments. The antibodies used for western blotting and IP were as follows: FLII antibody (sc-21716, Santa Cruz), PD-L1 antibody (ab205921, Abcam), HA antibody (\#2367, Cell Signaling Technology), YBX1 antibody (20339-1AP, Proteintech), anti-FLAG antibody (66008-2-Ig, 20543-1-AP, Proteintech), anti-GAPDH antibody (sc47724, Santa Cruz Biotechnology), Lamin A/C antibody (\#4777, Cell Signaling Technology), and Lamin A antibody (\#86846, Cell Signaling Technology).

\section{Direct protein binding by GST pull-down assays}

Direct binding of proteins in vitro was performed as previously described [22].

\section{Enzyme-linked immunosorbent assay (ELISA)}

The secretion of T cell cytokines was examined by a cytokine ELISA using a human or mouse ELISA kit (ExCell Bio) according to the manufacturers' recommendations. Enzyme activity was examined at 450 nm using an ELISA reader.

\section{Chromatin immunoprecipitation (ChIP) assays}

ChIP assays were performed according to protocols as previously described [24]. YBX1 and FLII antibodies were used to perform IP, and normal IgG was used as a negative control. Two percent of the original DNA was used as a positive input control.

\section{C4-2-R/T cell coculture model}

Peripheral blood mononuclear cells (PBMCs) from healthy people were segregated by Lymphoprep density gradient centrifugation (Accurate Chemical). PBMCs were plated at a density of $2 \times 10^{6}$ per well in six-well plates and stimulated with C4-2-R cell lysates, anti-CD28 $(2 \mu \mathrm{g} / \mathrm{ml})$ and anti-CD3e $(10 \mu \mathrm{g} / \mathrm{ml})$ for $48 \mathrm{~h}$ to facilitate $\mathrm{T}$ cell activation. The $\mathrm{T}$ cell activation protocols were provided by eBioscience (http://www.ebioscience.com/cell-type/t-cells.htm). C4-2-R cells were engineered to either stably express FLII or to stably express FLII and have YBX1 stably knocked down. Stimulated PBMCs were then harvested and purified by Lymphoprep density gradient centrifugation and cocultured with C4-2-R cells at a 10:1 ratio for $16 \mathrm{~h}$. The cocultured media were analyzed for TNF- $\alpha$, IFN-y, IL-2, IL-1 $\beta$, TGF- $\beta$ and IL-10 using ELISAs.

\section{RM-1 syngeneic C57BL/6 mouse model}

RM-1 cells were cultured, harvested, and suspended in PBS. For flank subcutaneous injections, a total of $0.1 \mathrm{ml}$ containing $2 \times 10^{6}$ cells was injected subcutaneously into the flanks of 6 - to 8-week-old C57BL/6 mice (Beijing HFK Bio-technology Co., Ltd). Ten days after injection, these mice were treated with enzalutamide. Tumor growth was evaluated. Tumor tissues were used for analysis of CD8+ T cells, myeloid-derived suppressor cells (MDSCs) and Treg subsets. The mouse serum was analyzed for IL-10, TNF- $\alpha$, TGF- $\beta$, and IFN- $\gamma$ with cytokine ELISAs. All the experimental and animal care protocols were 
approved by the Institutional Review Board of Tongji Medical College, Huazhong University of Science and Technology.

\section{Statistical analysis}

All data were analyzed using GraphPad Prism version 7 software. Normally distributed continuous variables were represented as the mean \pm standard error (SEM), and non-normally distributed continuous variables were represented as the median. For normally distributed variables, an independent Student's ttest was used to compare two groups. One-way ANOVA followed by Tukey's multiple comparison test was used to compare multiple groups. The correlation between variables with a normal distribution was evaluated using the Pearson correlation test, whereas nonnormally distributed variables were evaluated using the Spearman correlation test. $p<0.05$ was considered statistically significant.

\section{Results}

\section{FLII expression is negatively correlated with PD-L1}

Previous studies have shown that PD-L1 expression is upregulated in enzalutamide-resistant CRPC cells [25]. Consistent with previous studies, we also found that PD-L1 protein and mRNA levels were upregulated, while FLII protein and mRNA levels were down-regulated in enzalutamide-resistant C4-2 (C42-R) cells (Fig. 1a and Supplementary Fig. 1a-b. Our previous studies showed that FLII enhanced the sensitivity of CRPC cells to enzalutamide treatment [22]. To explore the mechanism, we reintroduced FLII into C4-2-R, C4-2 and PC-3 cells. As shown in Fig. 1a, FLIl downregulated the expression of PD-L1 protein in PCa cells. In contrast, knockdown of FLIl upregulated PD-L1 protein expression (Supplementary Fig. 1C. To evaluate the physiological correlation between FLII and PD-L1 expression, we performed a pooled in silico analysis of FLII and PD-L1 expression using multiple publicly available prostate cancer databases. As shown in Fig. 1b, FLII was negatively correlated with PD-L1 expression in all prostate cancer databases according to bioinformatics analysis. Next, we analyzed the correlation between FLII expression and the number of tumor-infiltrating lymphocytes (TILs) using a PCa TCGA dataset composed of 496 prostate cancer patient samples. The results showed that FLII expression was positively correlated with TILs, including B cells, CD8+ T cells, CD4+ T cells, macrophages, neutrophils, and dendritic cells (Fig. 1c).

To further validate the association between FLII and PD-L1 expression, we examined the expression levels of FLII, PD-L1 and CD8A (cytotoxic/suppressor T cell marker) using immunohistochemistry (IHC) in a prostate cancer tissue microarray (TMA). Consistent with our previous results, we found that FLII expression was negatively correlated with PD-L1 (Spearman rank correlation test, $r=-0.30, p=0.026$ ) and positively correlated with CD8A (Spearman rank correlation test, $r=0.42, p=0.0012$ ) (Fig. 1d). The protein expression levels of PD-L1 were significantly higher in PCa tissues $(n=59)$ than in normal tissues $(n=32)$, while the protein expression levels of FLII and CD8A showed the opposite relationships (Fig. 1d).

\section{FLII regulates the expression of PD-L1 via a YBX1-dependent signaling pathway}


Many studies have reported that YBX1 plays a role in multidrug resistance in cancer therapy [26-28]. An increase in the proportion of YBX1 entering the nucleus in multidrug-resistant cells has been confirmed [29]. Moreover, our previous study found that YBX1 transcriptionally regulated the expression of PD-L1 in doxorubicin-resistant HepG2 cells [24]. Therefore, we examined the expression of YBX1 in C4-2-R cells. As shown in Fig. 2a, the proportion of YBX1 entering the nucleus was significantly higher in C4-2-R cells than in parental cells, consistent with previous reports. Knockdown of YBX1 downregulated PD-L1 protein expression in C4-2-R and C4-2 cells (Fig. 2a and Supplementary Fig. 2a).

YBX1 has been previously reported to bind to the Y-box sequence (ATTGG) of the target gene promoter region [27]. To clarify the mechanism by which YBX1 regulates PD-L1 in PCa, we searched for the proximal promoter region of human PD-L1 (-1 - $1.5 \mathrm{~kb}$ upstream of the transcription start site (TSS)) and found a Y-box sequence in it (Fig. 2b). To uncover the transcriptional regulation of PD-L1 by YBX1, we first performed a luciferase (LUC) reporter assay. C4-2-R and PC3 cells were cotransfected with YBX1 overexpression plasmids and luciferase reporter plasmids containing three different sequences and $\mathrm{Y}$-box mutation sequence (AAAAA) of the PD-L1 promoter region. As shown in Fig. 2b, PD-L1 luciferase activity was higher in cells cotransfected with YBX1 plasmid/PD-L1-C-LUC (PD-L1-C-LUC: contains a sequence -1 $\sim-1.5 \mathrm{~kb}$ upstream of the TSS, containing the Y-box) than in cells cotransfected with YBX1 plasmid/PDL1-A-LUC (PD-L1-A-LUC: contains a sequence -1 -0.5 kb upstream of the TSS, lacking the Y-box), in cells cotransfected with YBX1 plasmid/PD-L1-B-LUC (PD-L1-B-LUC: contains a sequence -1 -1 kb upstream of the TSS, lacking the Y-box), and in cells cotransfected with YBX1 plasmid/PD-L1-M-LUC (PD-L1-MLUC: contains a sequence $-1 \sim-1.5 \mathrm{~kb}$ upstream of the TSS, containing the Y-box mutation). To further confirm the luciferase reporter assay results, chromatin immunoprecipitation (ChIP) experiments were performed in C4-2-R and PC3 cells (Fig. 2c) and indicated that YBX1 bound to the PD-L1 promoter region containing the Y-box region (P6: -1, $121 \mathrm{~kb} \sim-1,301 \mathrm{~kb}$ ) and activated transcription of PD-L1. Since FLII was negatively correlated with PD-L1 expression, we tried to detect whether FLII affects PD-L1 transcription and its mechanisms. We cotransfected FLII overexpression plasmid and PD-L1-C-LUC plasmid into the C4-2-R cells with or without stable knockdown of YBX1. As shown in Fig. 2d, overexpression of FLII in C4-2-R cells significantly decreased PD-L1 luciferase activity (line 5 vs line 6). However, overexpression of FLII in C4-2-R cells with stable knockdown of YBX1 did not enhance the FLIImediated decrease in PD-L1 luciferase activity (line 7 vs line 8) (Fig. 2d). Furthermore, we performed ChIP experiments with FLII antibody in C4-2-R cells. As shown in Supplementary Fig. 2b, FLII didn't bind to the PD-L1 promoter region. Moreover, overexpression of FLII inhibited YBX-1 binding to PD-L1 promoter region (Supplementary Fig. 2c). Similarly, overexpression of FLII significantly decreased PD-L1 protein in C4-2-R cells, while overexpression of FLII with stable knockdown of YBX1 did not enhance the FLIImediated decrease in PD-L1 protein expression in C4-2-R cells (Fig. 2e). These results indicate that FLII regulates the expression of PD-L1 via YBX1-dependent signaling pathway.

\section{FLII physically interacts with YBX1 and restricts YBX1 entry into the nucleus in C4-2-R cells}

Our previous studies indicate that FLII regulates the expression of PD-L1 via YBX1-dependent signaling pathway. However, the mechanism by which FLII regulates YBX1 signaling remains unclear. To explore 
the mechanism, we performed immunofluorescence experiments and found that FLII and YBX1 colocalized in the cytoplasm in C4-2 cells (Fig. 3a). We then performed immunoprecipitation experiments in human C4-2 cells and murine RM-1 cells and found that FLII and YBX1 bound to each other (Fig. 3b). To further determine the domains via which FLII bound to YBX1 and the domains via which YBX1 bound to FLII, we constructed truncated plasmids for FLII and YBX1 (Fig. 3c). HA-FLII wild-type (wt), HA-FLII-LRR (residues 1-450 aa), or HA-FLII-GLD (residues 451-1268 aa) with FLAG-YBX1 wt plasmids were cotransfected into HEK 293T cells. Flag-YBX1 was immunoprecipitated from 293T cell lysates with an anti-Flag antibody, and the FLII binding regions were detected with an anti-HA antibody in the anti-Flag pull-down lysates. We found that the HA-FLII wt and HA-FLII-GLD sequences coimmunoprecipitated with Flag-YBX1 (Fig. 3c). Then, Flag-YBX1 wt, Flag-YBX1-CSD (residues 51-128 aa), or Flag-YBX1-C-tem (residues 129-324 aa) with HA-FLII wt plasmids were cotransfected into HEK 293T cells. The IP results showed that the Flag-YBX1-C-term and Flag-YBX1 wt sequences coimmunoprecipitated with HA-FLII (Fig. 3c). In addition, we also performed direct protein binding with recombinant proteins. The results of GST pull down experiments showed that YBX1 could directly bind to FLII (Supplementary Fig. 3a). These results indicate that FLII physically interacts with YBX1.

Previous studies have reported an increase in the proportion of YBX1 entering the nucleus in multidrugresistant cells [29]. To explore whether the proportion of YBX1 entering the nucleus increased in C4-2-R cells, we performed immunofluorescence experiments. We transfected the Vector, HA-FLII-LRR and HAFLII-WT plasmids into the C4-2-R cells. FLII-LRR, the truncated fragment of FLII, did not bind to YBX1. As shown in Fig. 3d, FLII-WT, not FLII-LRR, sequestered YBX1 in the cytoplasm, suggesting that the interaction of FLII with YBX1 is required for the sequestration of YB-1 in the cytoplasm. Moreover, the reintroduction of FLII did not affect the expression of YBX1 protein in C4-2-R cells (Supplementary Fig. 3b). To further confirm the results of immunofluorescence staining, we performed cytoplasmic and nuclear protein extraction experiments for immunoblot analysis in C4-2 and C4-2-R cells. C4-2 and C4-2-R cells were transfected with HA-FLII or control plasmids. As shown in Fig. 3e, nuclear accumulation of YBX1 was reduced when HA-FLII plasmids were transfected, suggesting that FLII partly blocked the entry of YBX1 into the nucleus. Furthermore, a similar result was observed in C4-2 and C4-2-R cells with stable knockdown of FLII. As shown in Supplementary Fig. 3c, nuclear accumulation of YBX-1 was increased when FLII was knocked down in C4-2 and C4-2-R cells. Taken together, these results demonstrate that FLII antagonizes the nuclear accumulation of $\mathrm{YBX} 1$ in $\mathrm{C} 4-2-\mathrm{R}$ cells and may affect the transcriptional expression of YBX1 downstream target genes.

\section{FLII affects cytokine secretion in the tumor microenvironment in a C4-2-R/T cell coculture model}

High expression of PD-L1 in ENZ-resistant CRPC cells and the consequent reduction in dendritic cell infiltration into tumors might promote the immune evasion of CRPC cells [25], which indicates a potential association between ENZ treatment and immunoresistance. The PD-L1/PD-1 inhibitory pathway regulates $T$ cell activation and cytokine secretion in the tumor microenvironment [30,31]. Moreover, the secretion of cytokines in the tumor microenvironment is critical for many biological functions of $T$ cells [32]. Therefore, we assessed whether FLII-regulated PD-L1 expression affected cytokine secretion in the 
tumor microenvironment. We established a C4-2-R/T cell coculture model. T cell culture alone enhanced the secretion of TNF- - , IFN- $\gamma$, IL-2 and IL-1 $\beta$ and decreased the secretion of TGF- $\beta$ and IL-10 in the culture medium (Fig. 4a). Cocultured C4-2-R/T cells with stable FLII overexpression enhanced the secretion of TNF- $a$, IFN- $\gamma$ and IL-2 and decreased the secretion of TGF- $\beta$, IL-10 and IL-1 $\beta$ in culture medium (Fig. 4a). Furthermore, blocking PD-L1 activity with an anti-PD-L1 antibody abolished the FLII-mediated secretion of cytokines (Fig. 4a). The effect of the anti-PD-L1 antibody on cytokine secretion was similar to that of FLII overexpression. These results indicate that FLII regulates cytokine secretion in the tumor microenvironment by blocking a PD-L1-dependent pathway.

Our previous results demonstrated that FLII inhibited the transcriptional expression of PD-L1 by antagonizing the nuclear accumulation of YBX1. Therefore, we hypothesized that the YBX1 pathway might play a crucial role in FLII-mediated cytokine secretion in the tumor microenvironment. Unexpectedly, as shown in Fig. 4B, knockdown of YBX1 with a sh-YBX1 plasmid abolished FLII-mediated cytokine secretion in the C4-2-R/T cell coculture model. Furthermore, blocking PD-L1 activity with an anti-PD-L1 antibody abolished the sh-YBX1-mediated secretion of cytokines (Fig. 4b). The effect of the anti-PD-L1 antibody on cytokine secretion was similar to that of YBX1 knockdown. These results reveal that YBX1 regulates cytokine secretion by promoting a PD-L1-dependent pathway.

\section{YBX1 transcriptionally regulates PD-L1 expression in mouse PCa RM-1 cells}

We have previously demonstrated that YBX1 can transcriptionally regulate the expression of PD-L1 in human PCa cells. We hypothesized that YBX1 could also regulate the transcription of PD-L1 in murine PCa cells. To validate this hypothesis, we constructed FLII-overexpressing RM-1 cell and found that FLII downregulated PD-L1 protein expression (Fig. 5a). YBX1 knockdown with an sh-YBX1 plasmid significantly reduced PD-L1 protein expression and abolished the FLII-mediated decrease in PD-L1 protein expression in RM-1 cells (Fig. 5a). Luciferase (LUC) reporter assays indicated that FLIl overexpression significantly reduced PD-L1 luciferase activity, while YBX1 knockdown with an sh-YBX1 plasmid significantly reduced PD-L1 luciferase activity and abolished the FLII-mediated decrease in PD-L1 luciferase activity in RM-1 cells (Fig. 5b). To further confirm the results of luciferase reporter assays, chromatin immunoprecipitation (ChIP) experiments were performed in RM-1 cells (Fig. 5c) and indicated that YBX1 bound to the PD-L1 promoter region containing the Y-box region (P5: -1, $432 \mathrm{~kb} \sim-1,654 \mathrm{~kb}$ ) and activated transcription of PD-L1. To further verify the results of ChIP, RM-1 cells were cotransfected with YBX1 overexpression plasmids and luciferase reporter plasmids containing two different sequences of the PD-L1 promoter region. As shown in Fig. 5d, PD-L1 luciferase activity was higher in cells cotransfected with YBX1 plasmid/PD-L1-B-LUC (PD-L1-B-LUC: contains a sequence -1 -1.7 kb upstream of the TSS, containing the Y-box region) than in cells cotransfected with YBX1 plasmid/PD-L1-A-LUC (PDL1-A-LUC: contains a sequence -1 -1 kb upstream of the TSS, lacking the Y-box region).

\section{CD8+ T cells are required for FLIl overexpression-mediated antitumor immunity in RM-1 tumors}

The cells for tumor surveillance in the immune system are mainly $\mathrm{T}$ cells, which are essential components of the adaptive immune response [33]. When antigens on the surface of tumor cells are 
recognized by the $T$ cell receptor (TCR), the activated $C D 8+T$ cells attack tumor cells and kill them and are therefore named cytotoxic T cells (CTLs) [33]. Previous studies have reported that tumor regression after PD-1 blockade therapy requires pre-existing CD8+ T cells, which are negatively modulated via PDL1/PD-1-mediated adaptive immune inhibition [34]. To investigate the function of FLII, YBX1, PD-L1 and CD8+ T cells in vivo, we transplanted RM-1 cells with stable overexpression of FLII or YBX1 knockdown or vector into syngeneic C57BL/ 6 mice. These C57BL/ 6 mice were then treated with an anti-CD 8 antibody or an anti-PD-L1 antibody. Next, we examined the tumor volume in tumor-bearing mice. As shown in Fig. 5e, the groups with FLII overexpression, YBX1 knockdown or anti-PD-L1 had significantly reduced tumor volume compared to the control group, whereas CD8 antibody treatment disrupted the FLII-mediated tumor-suppressive effect (Tukey's multiple comparison test, $\mathrm{a}=0.05$; $\star *, \mathrm{P}<0.01$; ns, no significance). These results indicate that $\mathrm{CD} 8+\mathrm{T}$ cells are required for FLII overexpression-mediated function in tumorbearing C57BL/ 6 mice. Next, to verify whether FLII regulated anti-tumor immunity through YBX1, we examined the infiltration of CD8 + T cells, MDSCs and Tregs in RM-1 tumor tissues. As shown in SupplementaryFig. 4a-c, FLII overexpression or YBX1 knockdown increased the infiltration of CD8 + T cells and inhibited the infiltration of MDSCs and Tregs. These findings suggested that FLII regulated antitumor immunity through YBX1.

\section{FLII overexpression reverses ENZ resistance by modulating immunocyte production and cytokine secretion}

Our previous results demonstrated that FLII reduced the volume of RM-1 tumors. To explore the combined therapeutic effects of FLII and ENZ in prostate cancer, we injected RM-1 cells with stable overexpression of FLII or vector into syngeneic C57BL/ 6 mice. Then, these C57BL/ 6 mice were treated with ENZ. Surprisingly, ENZ treatment alone did not reduce the volume of RM-1 tumors, whereas the combination of FLII and ENZ significantly reduced the volume of RM-1 tumors (Tukey's multiple comparison test, $a=$ 0.05; **, $\mathrm{P}<0.01$; $* \mathrm{P}<0.05$; ns, no significance) (Fig. 6a). To explore the mechanism of RM-1 cell resistance to ENZ, we generated an ENZ resistance curve for RM-1 cells. We found that RM-1 cells were naturally resistant to ENZ. Moreover, we found that RM-1 cells naturally expressed AR-V7 via q-PCR (Supplementary Fig. 5a). Moreover, the combination treatment of FLII and ENZ significantly increased the proportion of CTLs in TILs in C57BL/6 mice (Supplementary Fig. 5b). This result indicated that FLII enhanced the sensitivity to ENZ treatment in ENZ-resistant RM-1 tumors. Flow cytometry analysis of RM1 tumor tissue showed that ENZ alone significantly increased PD-L1 expression, whereas combination treatment with FLII and ENZ significantly reduced PD-L1 expression (Tukey's multiple comparison test, a $=0.05 ; * \star \star, \mathrm{P}<0.001$, ns, no significance) (Fig. 6b). Myeloid-derived suppressor cells (MDSCs) and Tregs are the main components of the immunosuppressive tumor microenvironment (TME) [35]. To explore the mechanism by which FLII reverses immune evasion, we examined the infiltration of TILs, specifically, CD8+ T cells, MDSCs, and Tregs, in RM-1 tumor tissues. Consistent with the results of the PCa TCGA database analysis (Fig. 1C), FLII treatment increased the infiltration of CD8+ T cells and inhibited the infiltration of MDSCs and Tregs (Fig. 6ce-e). ENZ treatment alone did not affect the infiltration of CD8+ T cells, MDSCs and Tregs, whereas the combination treatment of FLII and ENZ significantly increased the infiltration of CD8+ T cells and suppressed the infiltration of MDSCs and Tregs (Tukey's multiple 
comparison test, $\mathrm{a}=0.05 ;{ }^{* \star \star}, \mathrm{P}<0.001$, ns, no significance) (Fig. 6ce-e). In addition, we injected RM-1 cells with stable knockdown of FLII or vector into syngeneic C57BL/6 mice. As shown in Supplementary Fig. 5c, knockdown of FLII in RM-1 tumors increased the tumor volume. Furthermore, knockdown of FLII decreased the production of CD8+ T cells and increased the production of MDSC and Tregs. These results demonstrated that FLII combated immune evasion by regulating immunocyte infiltration in the TME in ENZ-resistant RM-1 tumors. Our previous results indicated that FLII regulated the secretion of T cell cytokines via targeting the YBX1/PD-L1 pathway in a C4-2-R/T cell coculture model. Therefore, we also detected the secretion of $T$ cell cytokines in the serum of C57BL/6 mice. As shown in Fig. $\mathbf{6 f}$, ENZ treatment alone did not affect the secretion of TNF-a, IFN- $\gamma$, IL-10 and TGF- $\beta$ in C57BL/ 6 mouse serum. FLII treatment significantly increased the secretion of TNF- $\alpha$ and IFN- $\gamma$ and decreased the secretion of IL10 and TGF- $\beta$, and this effect was further enhanced by the combination treatment with ENZ and FLII (Tukey's multiple comparison test, $a=0.05 ; * \star \star, P<0.001$, ns, no significance) (Fig. 6f). Based on our in vitro and in vivo experimental results, we generated a schematic of the mechanisms of ENZ resistanceinduced immune evasion mediated by the FLII-YBX1-PD-L1 signaling axis (Fig. 6g).

\section{Discussion}

Immune checkpoint inhibitors targeting the PD-L1/PD-1 and CTLA-4 pathways have become a promising new and therapeutically efficacious strategy in the treatment of a variety of malignancies [36]. Blockade of PD-1 and CTLA-4 pathways reversed CD8+ TIL dysfunction and lead to tumor rejection in mice [37]. Furthermore, blocking the PD-1 and CTLA-4 pathways increased CD8+ and CD4+ T cell proliferation, antigen-specific cytokine secretion, Treg inhibition, and the upregulation of critical signaling pathway molecules for T cell function [37]. PD-L1 expression has been reported to be extremely low in prostate cancer [11]. Therefore, immune checkpoint inhibitors targeting PD-L1/PD-1 alone have not been able to achieve satisfactory clinical responses in prostate cancer. Previous studies have shown that the combination of immune checkpoint inhibitors with hormonal therapy in CRPC can enhance immune system responses and induce long-lasting clinical responses without obvious toxic side effects [14]. However, the specific mechanisms by which the combination of immune checkpoint inhibitors with hormonal therapy benefits patients with hormonal therapy resistance remain unclear.

Neo-endocrine therapy is the main clinical treatment modality for CRPC. However, neo-endocrine therapy resistance remains a serious clinical problem that compromises the therapeutic effect and ultimately leads to treatment failure in CRPC. Neo-endocrine therapy reagents for CRPC include abiraterone and enzalutamide. Recently, studies have confirmed the activation of the PD-L1/PD-1 pathway in CRPC that is resistant to neo-endocrine therapy, and this pathway activation leads to a decrease in tumor-specific $T$ cell activity, which potentially promotes the immune evasion of tumor cells [25]. Consistent with the above results, a small phase II clinical trial reported a rapid reduction in prostate-specific antigen (PSA) in $30 \%$ of patients with ENZ-resistant prostate cancer treated with the anti-PD-1 antibody pembrolizumab [14]. Therefore, there is some potential association between neo-endocrine therapy and immunoresistance in CRPC. However, it remains unclear what biomolecules regulate the PD-L1/PD-1 pathway or how neo-endocrine therapy modulates antitumor immunity and induces immunoresistance in 
ENZ-resistant CRPC. Y-box binding protein 1 (YBX1), a member of the cold-shock domain protein family, has been shown to induce chemotherapy-based immunoresistance in hepatocellular carcinoma [24]. Nuclear localization and increased expression of the transcription factor YBX1 regulate the transcriptional expression of multidrug resistance gene (MDR1), thereby inducing multidrug resistance in tumor cells [38]. Nevertheless, the crucial function of YBX1 in the neo-endocrine therapy-based immune evasion of CRPC remains unknown. In this study, we found that YBX1 and PD-L1 were associated with an immune evasion phenotype in ENZ-resistant CRPC. Our findings showed that YBX1 directly regulated the transcriptional expression of PD-L1 in human and mouse prostate cancer cells. Knockdown of YBX1 reduced PD-L1 expression in ENZ-resistant CRPC cells. Moreover, YBX1 regulated cytokine secretion in the tumor microenvironment by upregulating the expression of PD-L1. These results indicate that YBX1 plays a key role in neo-endocrine therapy resistance and induces tumor immunosuppression in the microenvironment and immune evasion in ENZ-resistant CRPC. Targeting the YBX1/PD-L1 signaling pathway, which could simultaneously reverse the immune evasion and multidrug resistance of ENZresistant CRPC cells, may benefit CRPC patients.

FLII protein, first found in Drosophila melanogaster, contains GLD and LRR domains. Our previous study found that FLII reversed enzalutamide resistance by promoting AR protein degradation and inhibiting AR nuclear accumulation [22]. Moreover, FLII inhibited the tumor cell growth, migration and invasion in PCa through AR-dependent pathways. High expression of FLII predicted a better prognosis and reversed ENZ resistance in CRPC. However, the roles and mechanisms of FLII in the ENZ resistance-induced immune evasion in CRPC remain unclear. Herein, we found that FLII was negatively correlated with PD-L1 expression and that low FLII expression predicted an immune-resistant phenotype in ENZ-resistant CRPC. FLII physically interacted with YBX1 and inhibited its nuclear localization in ENZ-resistant CRPC cells. FLII suppressed IFNY-induced, PD-L1-mediated CD8+ T cell apoptosis and modulated cytokine secretion in the tumor microenvironment by targeting the YBX1/PD-L1 pathway in ENZ-resistant CRPC cells and an ENZ$\mathrm{R} / \mathrm{T}$ cell coculture model. Furthermore, $\mathrm{CD} 8+\mathrm{T}$ cells are required for FLIl overexpression-mediated tumor suppression in CRPC. These findings indicate that FLII plays a key role in tumor cell immunoresistance and immune evasion in ENZ-resistant CRPC and that inhibition of the YBX1/PD-L1 signaling pathway may be possible by increasing the expression levels of FLII, thereby enhancing the therapeutic effect of strategies targeting neo-endocrine therapy-based immune evasion.

Blockade of the PD-L1/PD-1 pathway enhanced antitumor immunity and reduced the exhaustion of TILs in the tumor microenvironment by increasing the proliferation of effector CD8+ T cells and inhibiting Tregs and MDSCs, thereby yielding more powerful tumor control [39]. Increased infiltration of Tregs and MDSCs contributes to the immunosuppressive microenvironment of tumors [40]. Herein, combination therapy with FLII and enzalutamide was very effective in ENZ-resistant CRPC. This effect was likely a result of the decreased PD-L1 expression mediated by FLII in RM-1 tumor cells. The current findings suggest that PD-L1 expression is inversely correlated with TIL infiltration in the tumor microenvironment and that its upregulation contributes to tumor immunosuppression in ENZ-resistant CRPC. Although FLII reintroduction induced a modest increase in TIL infiltration, the number of MDSCs and Tregs was not significantly reduced in the tumor microenvironment after FLII reintroduction alone. Fortunately, the 
combination of FLII and enzalutamide significantly enhanced the efficiency of neo-endocrine therapy by regulating the infiltration of effector CD8+ T cells, Tregs, and MDSCs and cytokine secretion in RM-1 tumors.

\section{Conclusions}

In these studies, we found that FLII regulated the expression of PD-L1 via a YBX1-dependent signaling pathway. We recognized an important mechanism by which FLII regulated the PD-L1/PD-1 immune checkpoint signaling pathway through the YBX1 signaling axis in ENZ-resistant CRPC. Restoring FLII expression reversed neo-endocrine therapy resistance via partial blockade of the YBX1/PD-L1 signaling pathway in vitro and in vivo. The synergistic effect of this neo-endocrine therapy and immunotherapy was due to the proliferation of effector CD8+ T cells and the inhibition of Tregs and MDSCs in the tumor microenvironment. This study provides convincing support for a targeted therapy involving the functional interaction between the FLII and YBX1/PD-L1 signaling pathways to treat neo-endocrine therapy-resistant CRPC.

\section{Declarations}

\section{Ethics approval and consent to participate}

All experimental procedures were approved by the Human Ethics Committee of Huazhong University of Science and Technology.

\section{Consent for publication}

Not applicable.

\section{Availability of supporting data}

The rest of the results are in the supplementary data.

\section{Competing interests}

The authors state that they have no conflicts of interest.

\section{Funding}

This work was supported by grant from National Natural Sciences Foundation of China (81672524), Hubei Provincial Natural Science Foundation of China (2018CFA038), Science, Technology and Innovation Commission of Shenzhen Municipality (JCYJ20180305164838833), Fundamental Research Funds for the Central University (201kfyRCPY0049) and Wuhan Union Hospital Scientific Research Fund (Free Innovation Pre-Research Fund, Grant No. 000005001).

\section{Author's contributions}


Conception and design: K. Chen and Z. Tao; Acquisition of data (provided animals, acquired and managed patients, etc.): H. Ruan, L. Bao; Data analysis: H. Ruan, K. Chen and Z. Tao; Study supervision: K. Chen and Z. Tao

\section{Acknowledgements}

We thank the members of the Chen and Tao Lab for helpful discussions and suggestions. We are grateful to Huazhong University of Science and Technology for providing a good experimental platform.

\section{References}

1. Siegel RL, Miller KD, Jemal A: Cancer statistics, 2018. CA Cancer J Clin 2018, 68:7-30.

2. Bray F, Ferlay J, Soerjomataram I, Siegel RL, Torre LA, Jemal A: Global cancer statistics 2018: GLOBOCAN estimates of incidence and mortality worldwide for 36 cancers in 185 countries. $C A$ Cancer J Clin 2018, 68:394-424.

3. Zhou Y, Bolton EC, Jones JO: Androgens and androgen receptor signaling in prostate tumorigenesis. J Mol Endocrinol 2015, 54:R15-29.

4. Mazaris E, Tsiotras A: Molecular pathways in prostate cancer. Nephrourol Mon 2013, 5:792-800.

5. Drake CG: Prostate cancer as a model for tumour immunotherapy. Nat Rev Immuno/2010, 10:580593.

6. Drake CG, Jaffee E, Pardoll DM: Mechanisms of immune evasion by tumors. Adv Immuno/ 2006, 90:51-81.

7. Topalian SL, Hodi FS, Brahmer JR, Gettinger SN, Smith DC, McDermott DF, Powderly JD, Carvajal RD, Sosman JA, Atkins MB, et al: Safety, activity, and immune correlates of anti-PD-1 antibody in cancer. N Engl J Med 2012, 366:2443-2454.

8. Beer TM, Kwon ED, Drake CG, Fizazi K, Logothetis C, Gravis G, Ganju V, Polikoff J, Saad F, Humanski P, et al: Randomized, Double-Blind, Phase III Trial of Ipilimumab Versus Placebo in Asymptomatic or Minimally Symptomatic Patients With Metastatic Chemotherapy-Naive Castration-Resistant Prostate Cancer. J Clin Oncol 2017, 35:40-47.

9. Kwon ED, Drake CG, Scher HI, Fizazi K, Bossi A, van den Eertwegh AJ, Krainer M, Houede N, Santos R, Mahammedi $\mathrm{H}$, et al: Ipilimumab versus placebo after radiotherapy in patients with metastatic castration-resistant prostate cancer that had progressed after docetaxel chemotherapy (CA184-043): a multicentre, randomised, double-blind, phase 3 trial. Lancet Oncol 2014, 15:700-712.

10. Hansen AR, Massard C, Ott PA, Haas NB, Lopez JS, Ejadi S, Wallmark JM, Keam B, Delord JP, Aggarwal R, et al: Pembrolizumab for advanced prostate adenocarcinoma: findings of the KEYNOTE028 study. Ann Oncol 2018, 29:1807-1813.

11. Martin AM, Nirschl TR, Nirschl CJ, Francica BJ, Kochel CM, van Bokhoven A, Meeker AK, Lucia MS, Anders RA, DeMarzo AM, Drake CG: Paucity of PD-L1 expression in prostate cancer: innate and adaptive immune resistance. Prostate Cancer Prostatic Dis 2015, 18:325-332. 
12. Bilusic M, Madan RA, Gulley JL: Immunotherapy of Prostate Cancer: Facts and Hopes. Clin Cancer Res 2017, 23:6764-6770.

13. O'Sullivan Coyne G, Madan RA, Gulley JL: Nivolumab: promising survival signal coupled with limited toxicity raises expectations. J Clin Oncol 2014, 32:986-988.

14. Graff JN, Alumkal JJ, Drake CG, Thomas GV, Redmond WL, Farhad M, Cetnar JP, Ey FS, Bergan RC, Slottke R, Beer TM: Early evidence of anti-PD-1 activity in enzalutamide-resistant prostate cancer. Oncotarget 2016, 7:52810-52817.

15. Mateo J, Carreira S, Sandhu S, Miranda S, Mossop H, Perez-Lopez R, Nava Rodrigues D, Robinson D, Omlin A, Tunariu N, et al: DNA-Repair Defects and Olaparib in Metastatic Prostate Cancer. N Eng/ J Med 2015, 373:1697-1708.

16. Archer SK, Behm CA, Claudianos C, Campbell HD: The flightless I protein and the gelsolin family in nuclear hormone receptor-mediated signalling. Biochem Soc Trans 2004, 32:940-942.

17. Kwiatkowski DJ: Functions of gelsolin: motility, signaling, apoptosis, cancer. Curr Opin Cell Bio/1999, 11:103-108.

18. Wu L, Chen H, Zhu Y, Meng J, Li Y, Li M, Yang D, Zhang P, Feng M, Tong X: Flightless I homolog negatively regulates ChREBP activity in cancer cells. Int J Biochem Cell Biol 2013, 45:2688-2697.

19. Lee YH, Stallcup MR: Interplay of Fli-I and FLAP1 for regulation of beta-catenin dependent transcription. Nucleic Acids Res 2006, 34:5052-5059.

20. Choi JS, Choi SS, Kim ES, Seo YK, Seo JK, Kim EK, Suh PG, Choi JH: Flightless-1, a novel transcriptional modulator of PPARgamma through competing with RXRalpha. Cell Signal 2015, 27:614-620.

21. Lee YH, Campbell HD, Stallcup MR: Developmentally essential protein flightless I is a nuclear receptor coactivator with actin binding activity. Mol Cell Biol 2004, 24:2103-2117.

22. Wang T, Song W, Chen Y, Chen R, Liu Z, Wu L, Li M, Yang J, Wang L, Liu J, et al: Flightless I Homolog Represses Prostate Cancer Progression through Targeting Androgen Receptor Signaling. Clin Cancer Res 2016, 22:1531-1544.

23. Ruan H, Yang H, Wei H, Xiao W, Lou N, Qiu B, Xu G, Song Z, Xiao H, Liu L, et al: Overexpression of SOX4 promotes cell migration and invasion of renal cell carcinoma by inducing epithelialmesenchymal transition. Int J Oncol 2017, 51:336-346.

24. Tao Z, Ruan H, Sun L, Kuang D, Song Y, Wang Q, Wang T, Hao Y, Chen K: Targeting the YB-1/PD-L1 Axis to Enhance Chemotherapy and Antitumor Immunity. Cancer Immunol Res 2019, 7:1135-1147.

25. Bishop JL, Sio A, Angeles A, Roberts ME, Azad AA, Chi KN, Zoubeidi A: PD-L1 is highly expressed in Enzalutamide resistant prostate cancer. Oncotarget 2015, 6:234-242.

26. Tsofack SP, Garand C, Sereduk C, Chow D, Aziz M, Guay D, Yin HH, Lebel M: NONO and RALY proteins are required for YB-1 oxaliplatin induced resistance in colon adenocarcinoma cell lines. Mol Cancer 2011, 10:145. 
27. Kuwano M, Shibata T, Watari K, Ono M: Oncogenic Y-box binding protein-1 as an effective therapeutic target in drug-resistant cancer. Cancer Sci 2019, 110:1536-1543.

28. Mo D, Fang H, Niu K, Liu J, Wu M, Li S, Zhu T, Aleskandarany MA, Arora A, Lobo DN, et al: Human Helicase RECQL4 Drives Cisplatin Resistance in Gastric Cancer by Activating an AKT-YB1-MDR1 Signaling Pathway. Cancer Res 2016, 76:3057-3066.

29. Ohga T, Koike K, Ono M, Makino Y, Itagaki Y, Tanimoto M, Kuwano M, Kohno K: Role of the human $Y$ box-binding protein YB-1 in cellular sensitivity to the DNA-damaging agents cisplatin, mitomycin C, and ultraviolet light. Cancer Res 1996, 56:4224-4228.

30. Blank C, Gajewski TF, Mackensen A: Interaction of PD-L1 on tumor cells with PD-1 on tumor-specific T cells as a mechanism of immune evasion: implications for tumor immunotherapy. Cancer Immunol Immunother 2005, 54:307-314.

31. Brown JA, Dorfman DM, Ma FR, Sullivan EL, Munoz O, Wood CR, Greenfield EA, Freeman GJ: Blockade of programmed death-1 ligands on dendritic cells enhances $T$ cell activation and cytokine production. J Immunol 2003, 170:1257-1266.

32. Hoeppli RE, Wu D, Cook L, Levings MK: The environment of regulatory T cell biology: cytokines, metabolites, and the microbiome. Front Immunol 2015, 6:61.

33. Igney FH, Krammer PH: Immune escape of tumors: apoptosis resistance and tumor counterattack. $J$ Leukoc Biol 2002, 71:907-920.

34. Tumeh PC, Harview CL, Yearley JH, Shintaku IP, Taylor EJ, Robert L, Chmielowski B, Spasic M, Henry G, Ciobanu V, et al: PD-1 blockade induces responses by inhibiting adaptive immune resistance. Nature 2014, 515:568-571.

35. Lindau $D$, Gielen $P$, Kroesen $M$, Wesseling $P$, Adema GJ: The immunosuppressive tumour network: myeloid-derived suppressor cells, regulatory T cells and natural killer T cells. Immunology 2013, 138:105-115.

36. Baksh K, Weber J: Immune checkpoint protein inhibition for cancer: preclinical justification for CTLA4 and PD-1 blockade and new combinations. Semin Oncol 2015, 42:363-377.

37. Duraiswamy J, Kaluza KM, Freeman GJ, Coukos G: Dual blockade of PD-1 and CTLA-4 combined with tumor vaccine effectively restores T-cell rejection function in tumors. Cancer Res 2013, 73:35913603.

38. Bargou RC, Jurchott K, Wagener C, Bergmann S, Metzner S, Bommert K, Mapara MY, Winzer KJ, Dietel $M$, Dorken B, Royer HD: Nuclear localization and increased levels of transcription factor YB-1 in primary human breast cancers are associated with intrinsic MDR1 gene expression. Nat Med 1997, 3:447-450.

39. Duraiswamy J, Freeman GJ, Coukos G: Therapeutic PD-1 pathway blockade augments with other modalities of immunotherapy T-cell function to prevent immune decline in ovarian cancer. Cancer Res 2013, 73:6900-6912.

40. Mauge L, Terme M, Tartour E, Helley D: Control of the adaptive immune response by tumor vasculature. Front Oncol 2014, 4:61. 
a
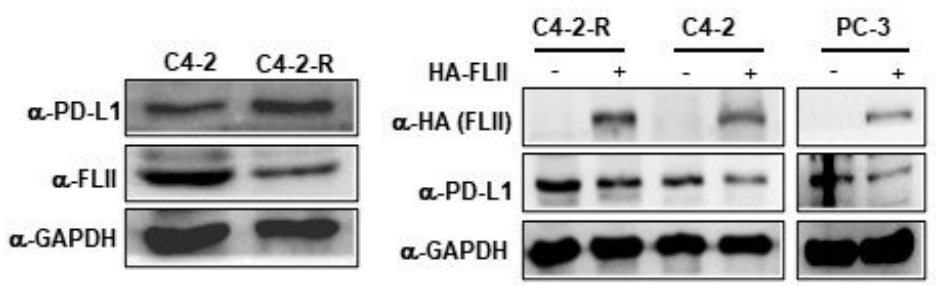

Figure. 1
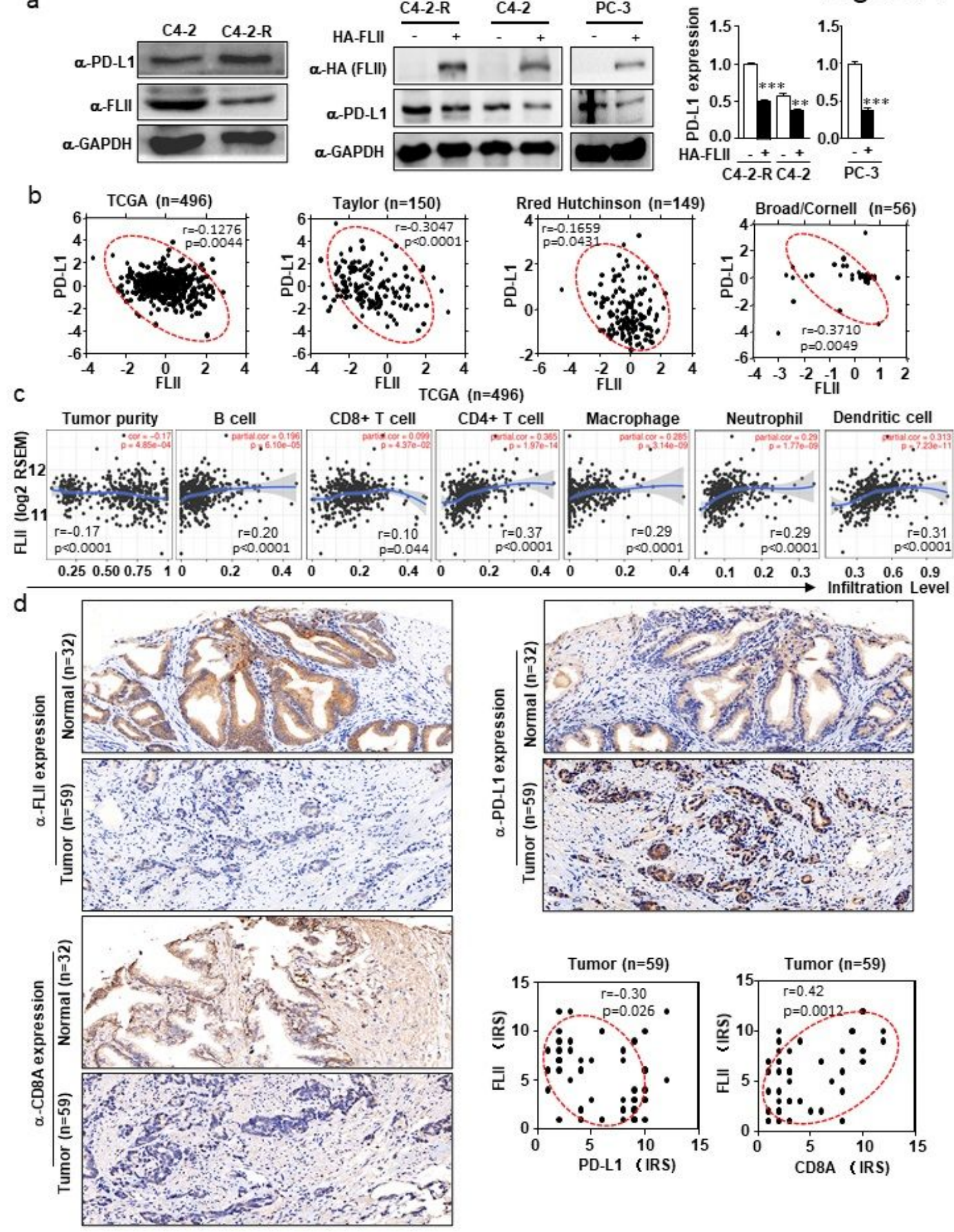

\section{Figure 1}

FLII expression is negatively correlated with PD-L1 in PCa. (a) Western blotting analysis showed that PDL1 was upregulated and FLII was downregulated in C4-2-R cells, and overexpression of FLII reduced PDL1 expression in PCa cells. The bar graph represents the quantification of PD-L1 protein expression. The 
error bars indicate the mean \pm SEM of three independent assays (Student's t-test, $\star \star \star, ~ P<0.001, \star \star, ~ P<$ 0.01) (b) FLII was negatively correlated with PD-L1 expression in human prostate cancer according to an analysis of publicly available cancer database data (TCGA, Taylor, Fred Hutchinson, and Broad/Cornell) (assessed with the Pearson rank correlation test). (c) The correlation between FLII expression and the number of tumor-infiltrating lymphocytes (TILs) was determined with a PCa TCGA database composed of 496 prostate cancer patient samples. (assessed with the Pearson rank correlation test). (d) FLII, PD-L1 and CD8A protein levels were analyzed using a PCa tissue microarray (TMA) containing 59 PCa tissues and 32 normal tissues. Representative IHC staining images for FLII, PD-L1 and CD8A are displayed. FLII and CD8A were significantly lower in tumor tissues than in normal tissues, whereas the expression of PDL1 was the opposite. FLII was negatively correlated with the expression of PD-L1 (Spearman rank correlation test, $r=-0.30, p=0.026$ ) and positively correlated with CD8A expression (Spearman rank correlation test, $r=0.42, p=0.0012$ ). 
Figure. 2

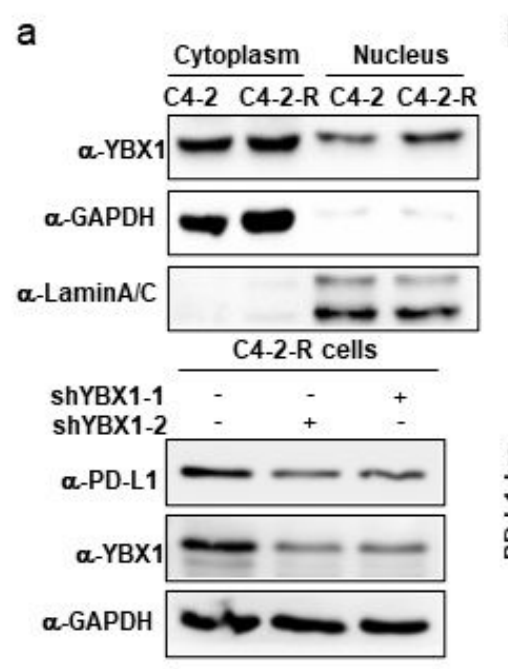

b

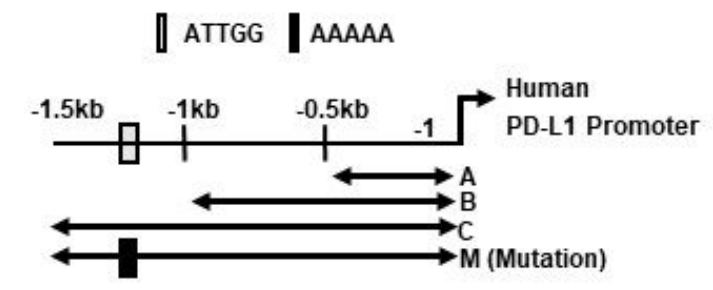

C
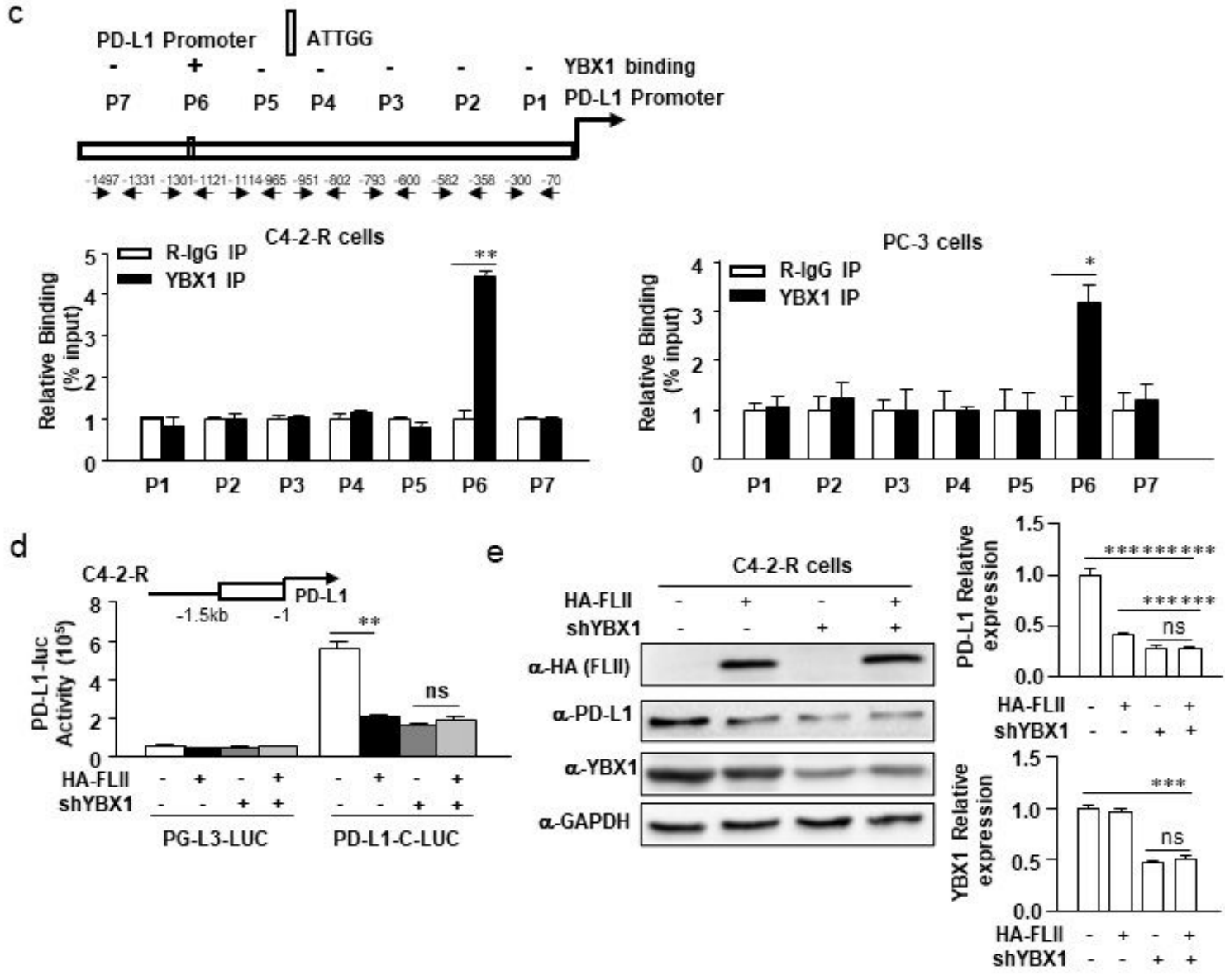

Figure 2

FLII regulates the expression of PD-L1 via a YBX1-dependent signaling pathway. (a) Cytoplasmic and nuclear expression of YBX1 was analyzed by western blot in C4-2 and C4-2-R cells. PD-L1 levels were reduced in C4-2-R cells with stable YBX1 knockdown. (b) The human PD-L1 promoter regions (-1 - $1.5 \mathrm{~kb})$ were incorporated into four luciferase reporters (PD-L1-LUC-A, B, C, M). YBX1 increased the activity of the PD-L1 luciferase reporter gene in C4-2-R and PC3 cells that contained the Y-box sequence (ATTGG). The 
error bars indicate the mean \pm SEM of three independent assays (Student's t-test, $* \star \star, P<0.001, * \star, P<$ 0.01, ns, no significance) (c) The human PD-L1 promoter region (-1 -1.5 kb) was divided into seven different segments. ChIP analysis was used to study the potential binding of YBX1 in the PD-L1 promoter region in C4-2-R and PC3 cells. YBX1 bound to the designated regions (P6: -1121 -1301 kb, containing the Y-box sequence) of the PD-L1 promoter. ChIP assays were performed using an anti-YBX1 antibody. The error bars indicate the mean \pm SEM of three independent assays (Student's t-test, $\star \star, P<0.01, *, P<$ 0.05). (d) FLII reduced the activity of the PD-L1 luciferase reporter gene in C4-2-R cells, and this effect was abolished by YBX1 knockdown. The error bars indicate the mean \pm SEM of three independent assays (Student's t-test, **, P $<0.01$, ns, no significance). (e) FLII reduced the protein levels of PD-L1 expression in C4-2-R cells, and this effect was abolished by YBX1 knockdown. The bar graph represents the quantification of PD-L1 and YBX1 protein expression. The error bars indicate the mean \pm SEM of three independent assays (Student's t-test, $\star \star \star, ~ P<0.001, n s$, no significance) 
a

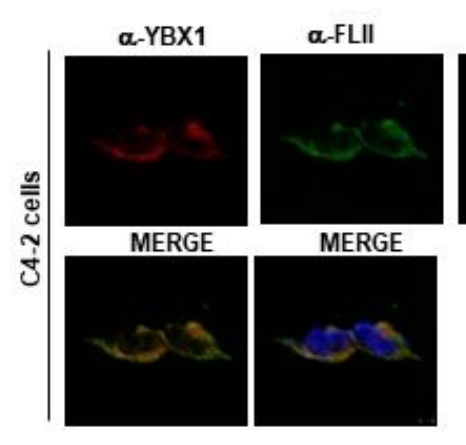

b

Figure. 3

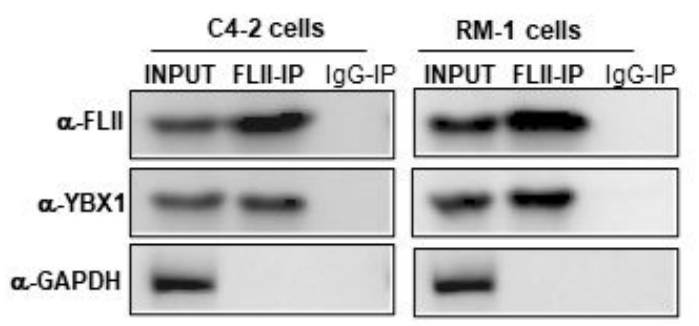

c
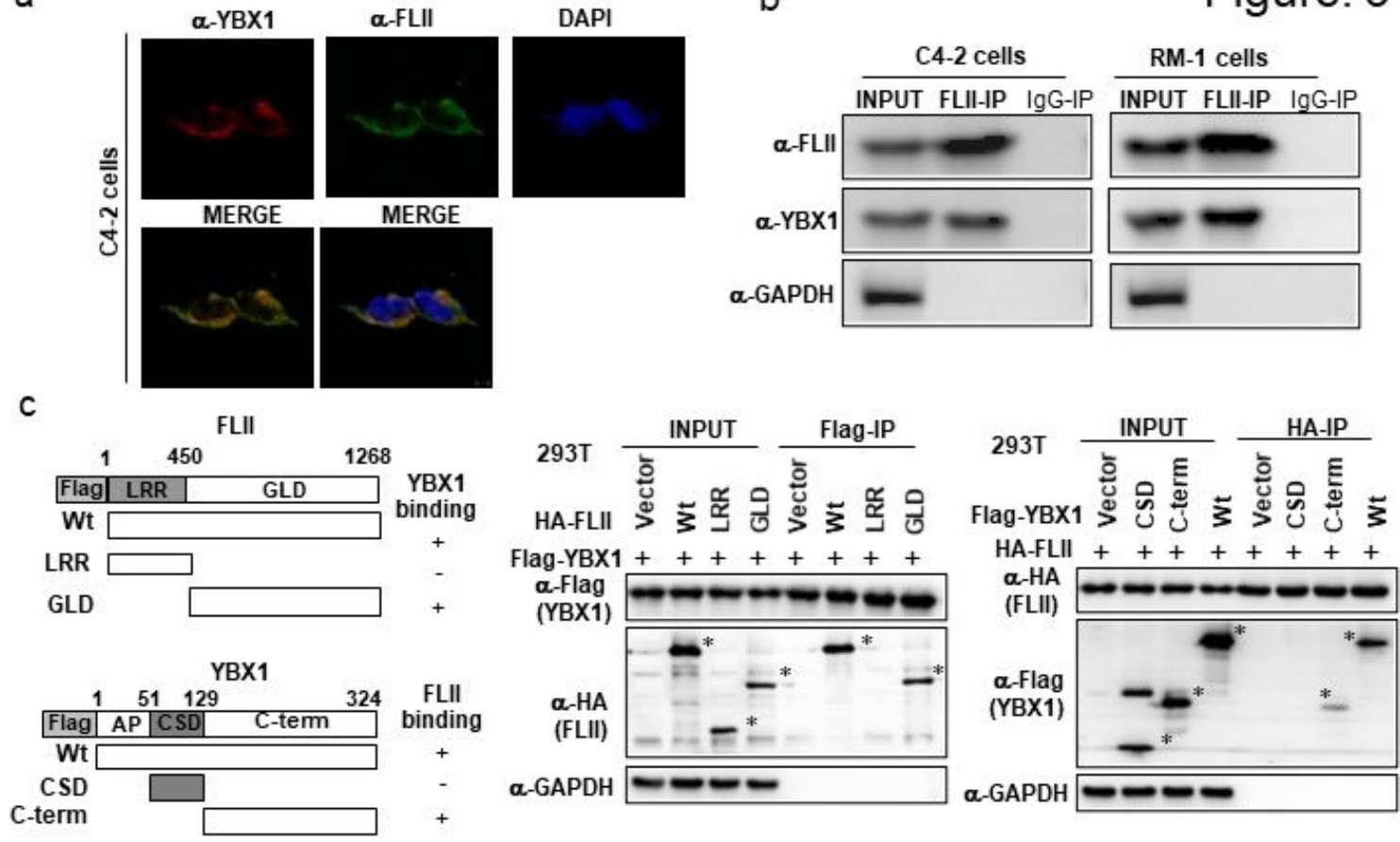

d
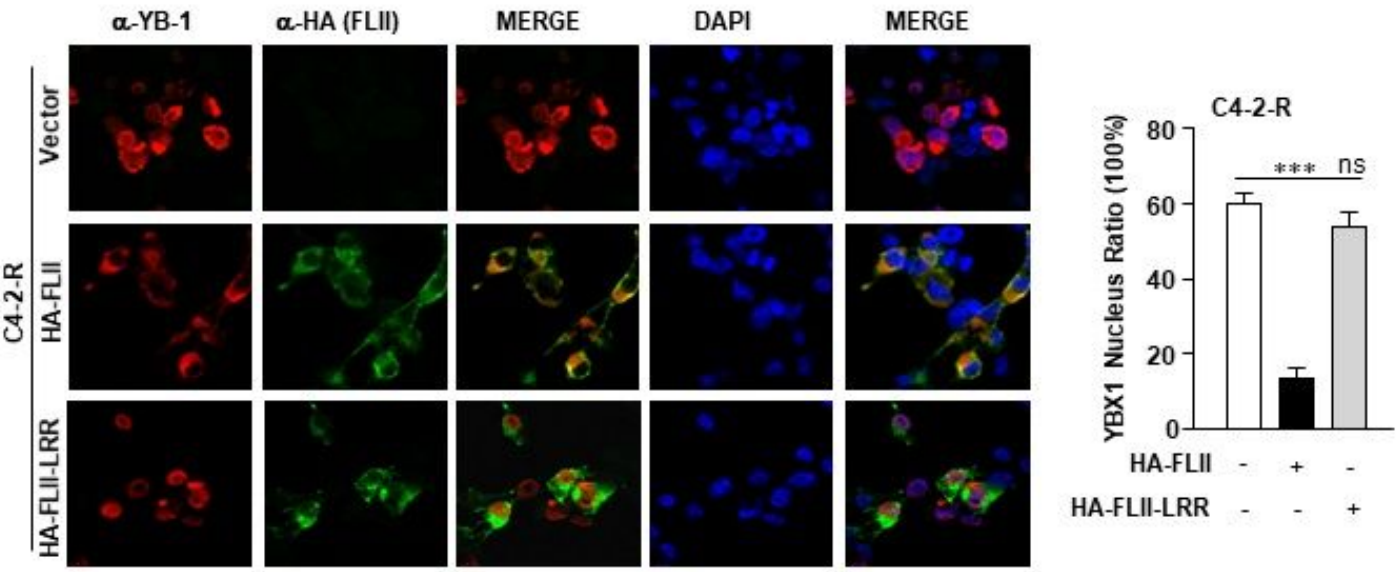

e

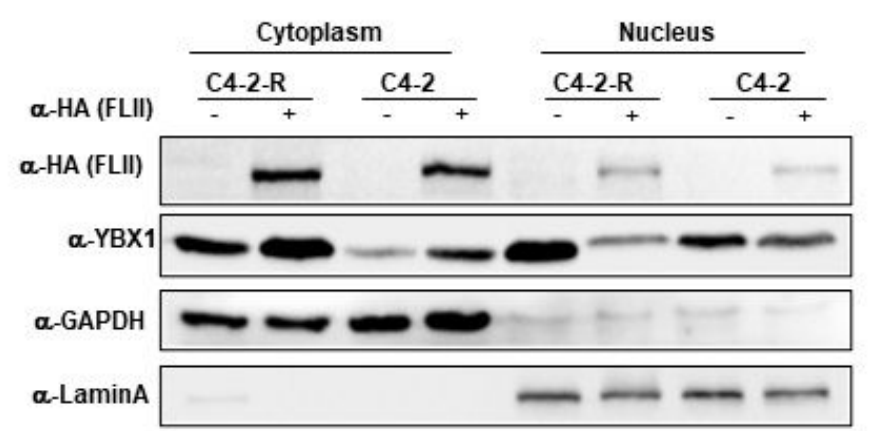

\section{Figure 3}

FLII physically interacts with YBX1 and restricts YBX1 entry into the nucleus in PCa cells. (a) Immunofluorescence (IF) staining showed that FLII and YBX1 colocalized in the cytoplasm of C4-2 cells. (b) Immunoprecipitation (IP) experiments revealed that FLII protein bound to YBX1 protein in C4-2 and RM-1 cells. (c) Schematic representation of the FLII and YBX1 truncated plasmid vectors. IP-western blot analysis was performed using 293T cells cotransfected with plasmid vectors encoding Flag-tagged YBX1 
and HA-tagged FLII truncated plasmid vectors. IP-western blot analysis was performed using 293T cells cotransfected with plasmid vectors encoding HA-tagged FLII and Flag-tagged YBX1 truncated plasmid vectors. (d) The expression and localization of FLII, FLII-LRR and YBX1 were determined by IF staining analysis in C4-2-R cells stably overexpressing FLII, FLII-LRR or vector. The error bars indicate the mean \pm SEM of three independent assays (Student's t-test, $\star \star \star, ~ P<0.001$, ns, no significance). (e) Western blot analysis of the cytoplasmic and nuclear expression of FLII and YBX1 in C4-2-R (vector and HA-FLII) and C4-2 (vector and HA-FLII) cells.

a
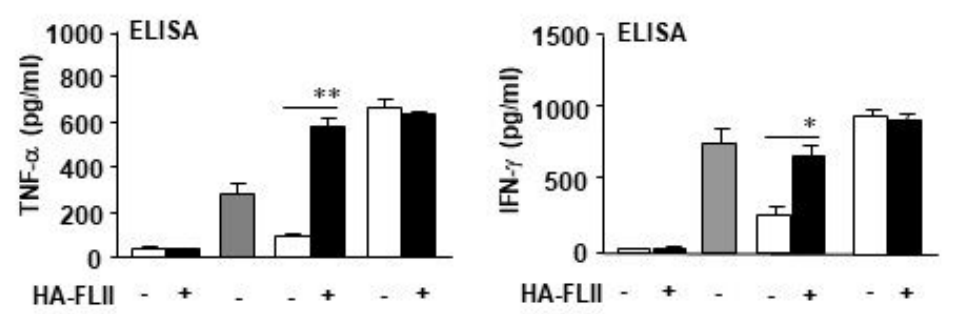

Fig. 4
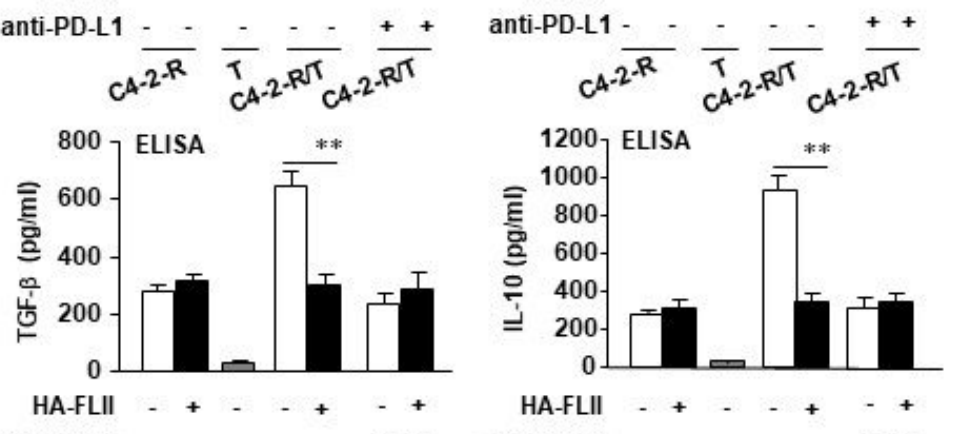

anti-PD-L1

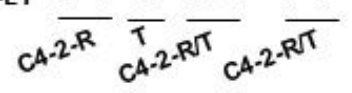

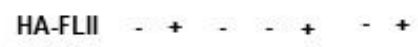

anti-PD-L1 - $-\ldots+\ldots+$

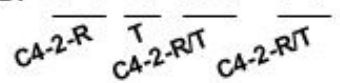

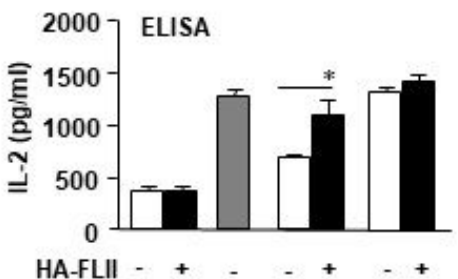

b
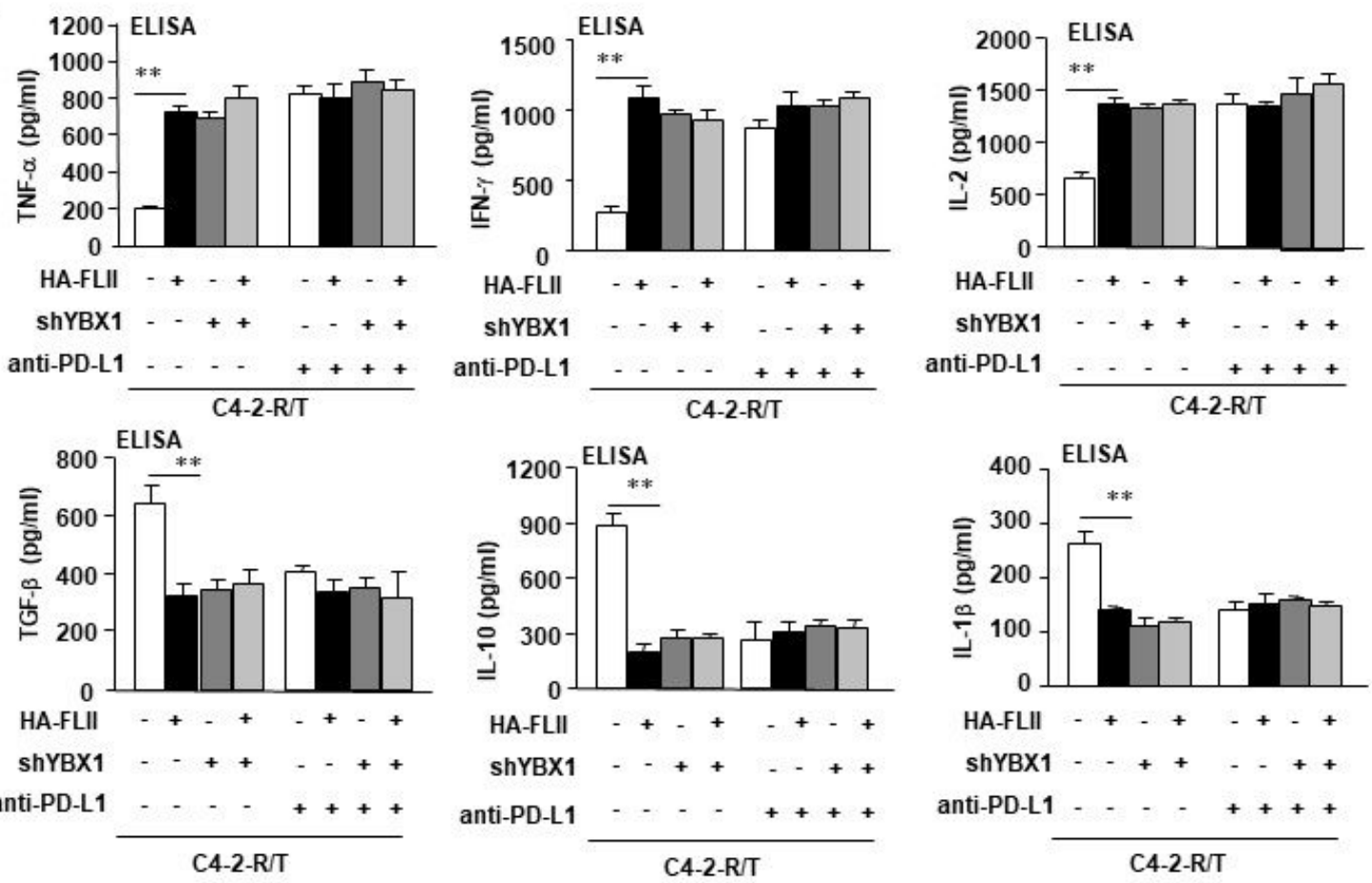


\section{Figure 4}

FLII affects cytokine secretion and T cell apoptosis in the tumor microenvironment in a C4-2-R/T cell coculture model. (a) C4-2-R cells stably overexpressing FLII or vector were treated with or without an antiPD-L1 antibody. After $24 \mathrm{~h}, \mathrm{~T}$ cells were subsequently cocultured with mitomycin C-treated C4-2-R cells for $24 \mathrm{~h}$. Cocultured media were analyzed for TNF- $\alpha$, IFN- - , IL-2, TGF- $\beta$, IL-10 and IL-1 $\beta$ by cytokine ELISAs. The error bars indicate the mean \pm SEM of three independent assays (Student's t-test, **, $\mathrm{P}<0.01, *, \mathrm{P}<$ 0.05). (b) C4-2-R cells with the indicated intervention were cocultured with $T$ cells. These cocultured cells were treated with or without an anti-PD-L1 antibody. Cocultured media were analyzed for TNF-a, IFN- $y$, IL2 , TGF- $\beta$, IL-10 and IL-1 $\beta$ by cytokine ELISAs. The error bars indicate the mean \pm SEM of three independent assays (Student's t-test, $* \star, P<0.01$ ). The error bars indicate the mean \pm SEM of three independent assays (Student's t-test, $\star \star, P<0.01$ ). 
Figure. 5

a

b
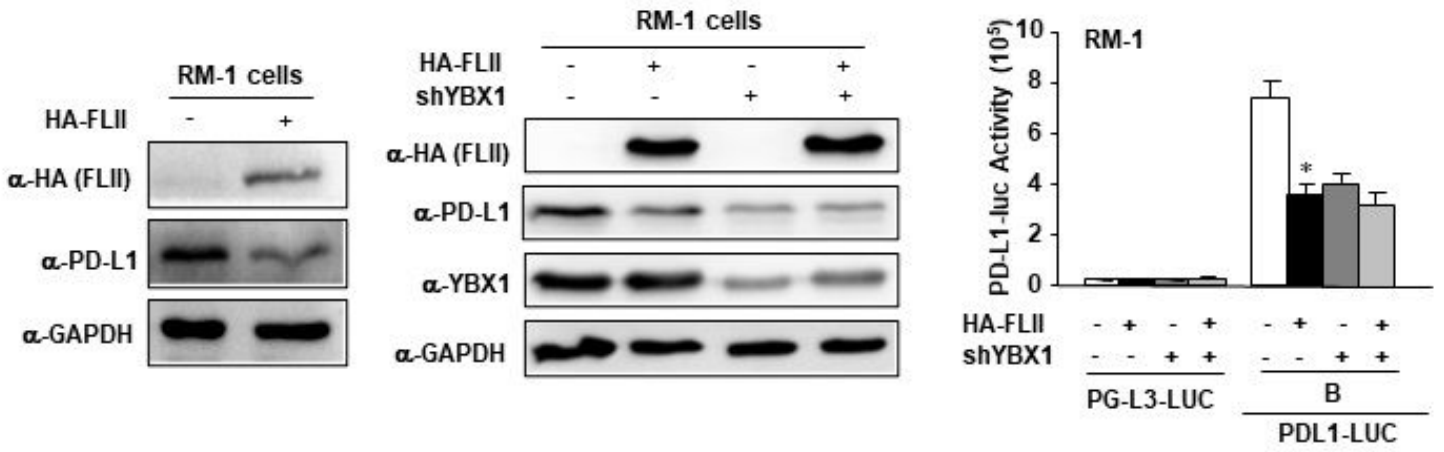

C

d
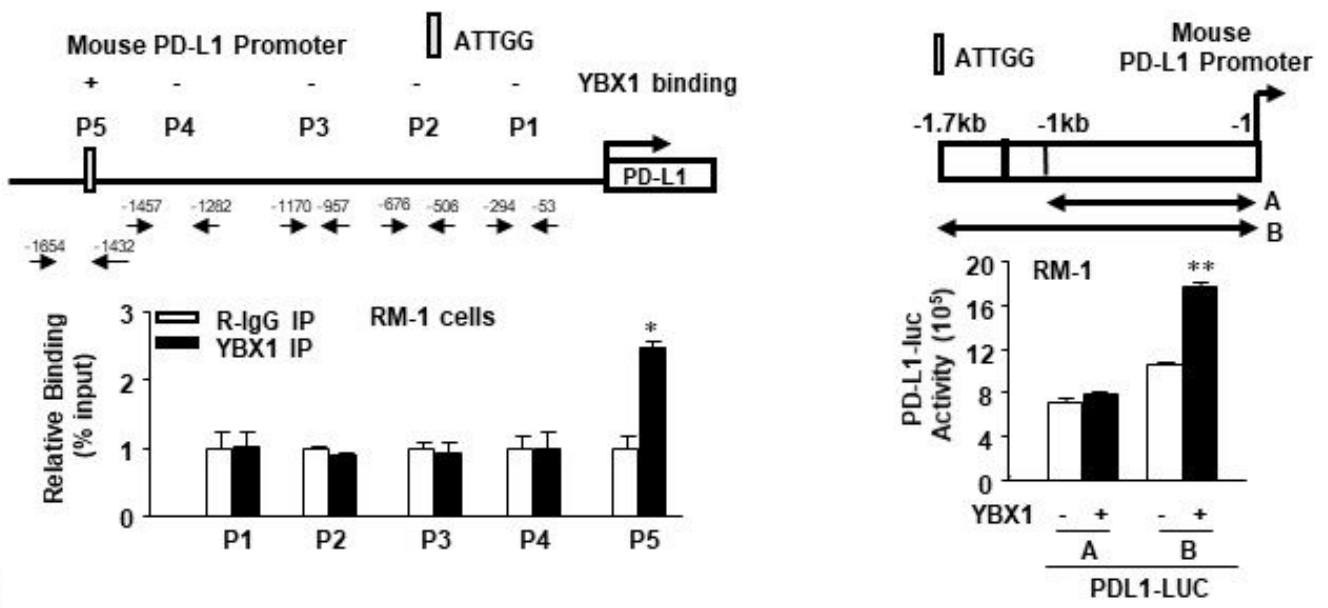

e
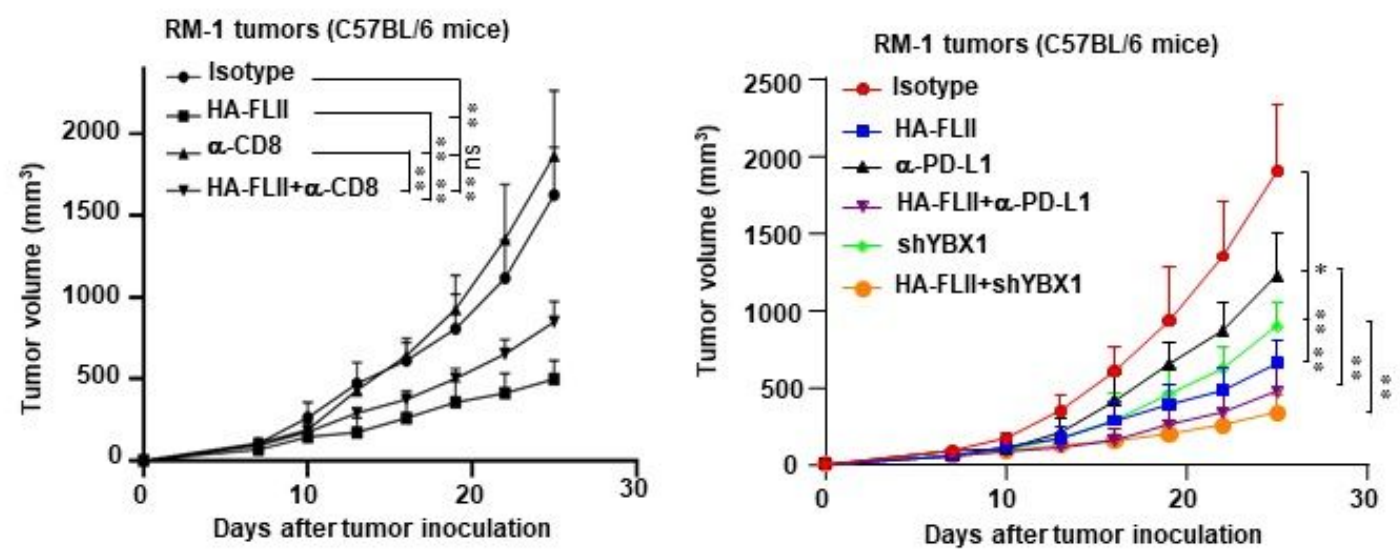

Figure 5

YBX1 transcriptionally regulates PD-L1 expression in mouse PCa RM-1 cells. (a) Western blot analysis showed that FLII reduced the protein levels of PD-L1 expression in mouse PCa RM-1 cells, and this effect was abolished by YBX1 knockdown. (b) FLII reduced the activity of the PD-L1 luciferase reporter gene in RM-1 cells, and this effect was abolished by YBX1 knockdown. The error bars indicate the mean \pm SEM of three independent assays (Student's t-test, *, P < 0.05). (c) The mouse PD-L1 promoter region (-1 -1.7 kb) 
was divided into five different segments. ChIP analysis was used to study the potential binding of YBX1 in the PD-L1 promoter region in RM-1 cells. YBX1 bound to the designated regions (P5: -1432 -1654 kb, containing the Y-box sequence) of the PD-L1 promoter. The error bars indicate the mean \pm SEM of three independent assays (Student's t-test, *, P< 0.05). (d) The mouse PD-L1 promoter regions $(-1 \sim-1.7 \mathrm{~kb})$ were incorporated into two luciferase reporter genes. YBX1 increased the activity of the PD-L1 luciferase reporter gene in RM-1 cells that contained the Y-box sequence (ATTGG). The error bars indicate the mean \pm SEM of three independent assays (Student's t-test, $\star \star, P<0.01$ ). (e) RM-1 cells stably overexpressing FLII or vector were seeded into C57BL/ 6 mice. These mice were treated with or without anti-CD8 or PD-L1 antibody. RM-1 cells stably overexpressing FLII/YBX1 knockdown or YBX1 knockdown were seeded into $\mathrm{C} 57 \mathrm{BL} / 6$ mice, and the tumor volume was measured over time. The error bars indicate the mean $\pm \mathrm{SEM}$ of three independent assays ( $n=12$ mice/group) (one-way ANOVA followed by Tukey's multiple comparison test, $a=0.05 ; \star \star, ~ P<0.01 ; *, P<0.05$, ns, no significance). 


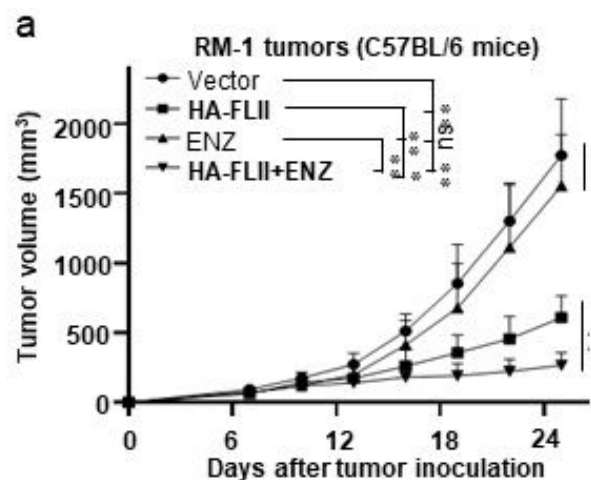

C
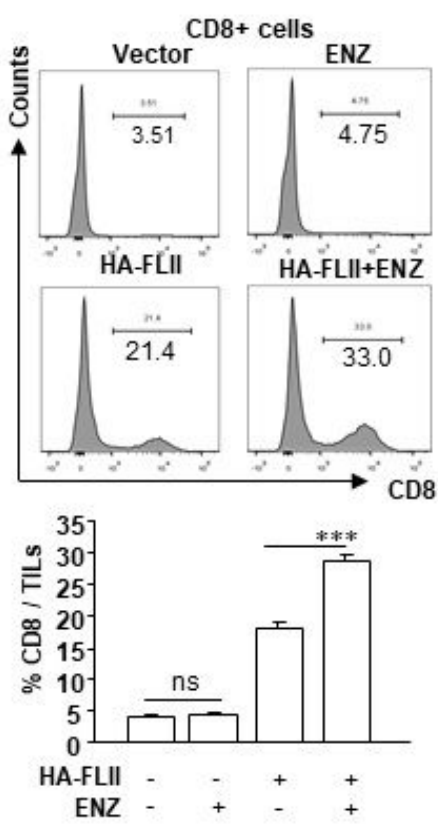

d
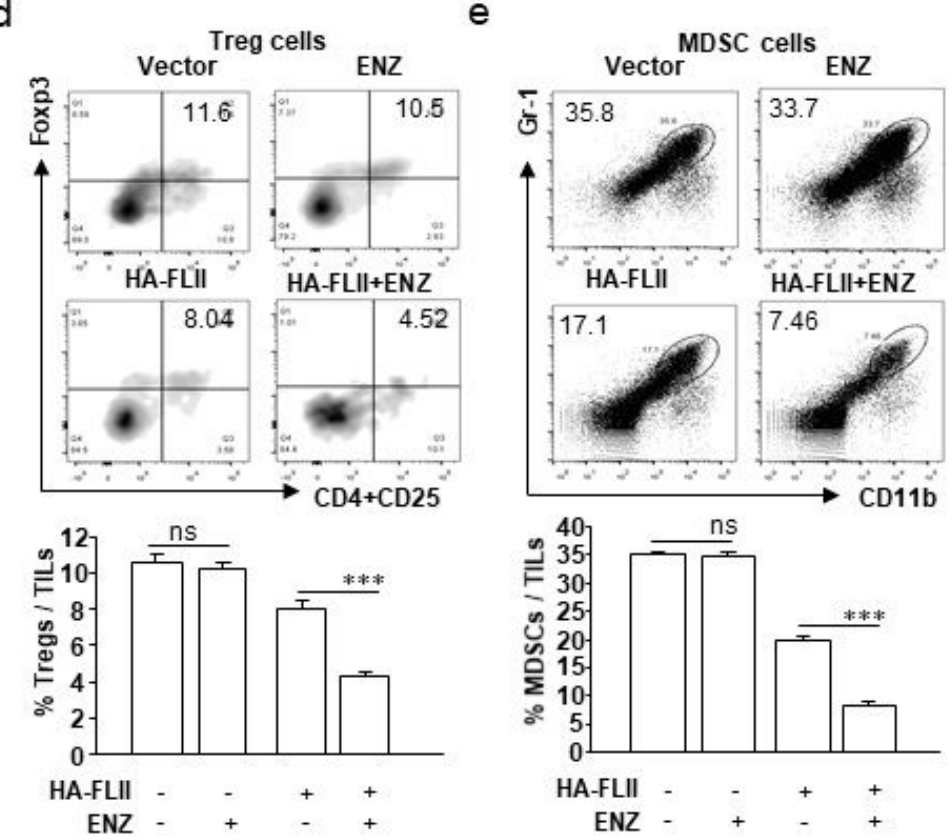

f
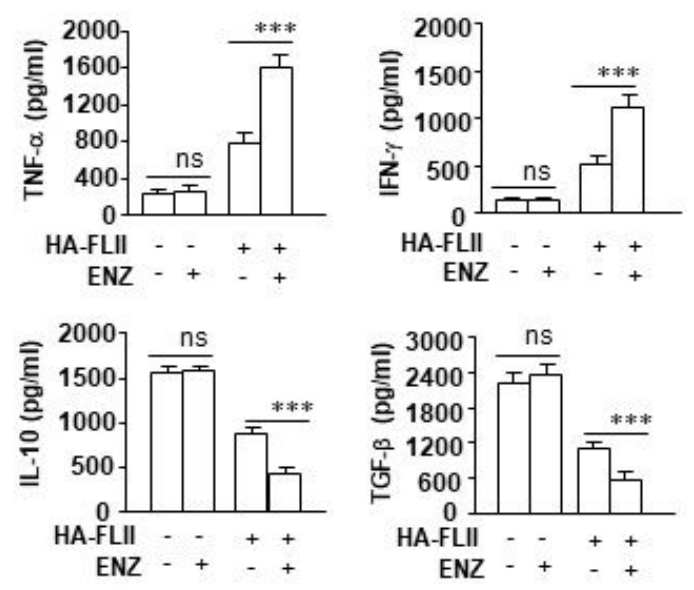

g

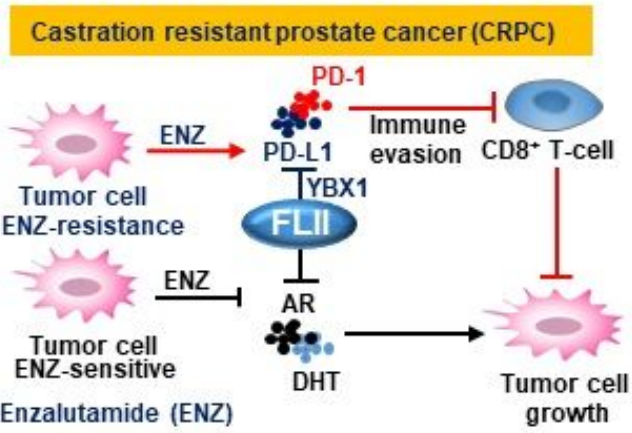

Figure 6

FLII overexpression enhances ENZ treatment efficacy by modulating immunocyte production and cytokine secretion. RM-1 cells stably overexpressing FLII or vector were seeded into C57BL/6 mice. These mice were treated with ENZ or vector. (a) RM-1 tumor volume was measured over time. The error bars indicate the mean \pm SEM of three independent assays ( $n=12$ mice/group) (one-way ANOVA followed by Tukey's multiple comparison test, $\mathrm{a}=0.05$; **, $\mathrm{P}<0.01$; *, $\mathrm{P}<0.05$; ns, no significance). (b) Tumor cell- 
surface PD-L1 expression was examined by FACS analysis in RM-1 tumor tissues. The error bars indicate the mean \pm SEM of three independent assays ( $n=12$ mice/group) (one-way ANOVA followed by Tukey's multiple comparison test, $a=0.05 ; * \star \star, P<0.001$, ns, no significance). (c-e) CD8+ T cells, Tregs and MDSCs from the tumors of RM-1 tumor-bearing mice were isolated and counted via FACS analysis. The error bars indicate the mean \pm SEM of three independent assays ( $n=12$ mice/group) (one-way ANOVA followed by Tukey's multiple comparison test, $a=0.05$; $* \star \star, P<0.001$, ns, no significance). (f) Circulating serum from C57BL/ 6 mice was analyzed for TNF-a, IFN- $\gamma$, IL-10 and TGF- $\beta$ by cytokine ELISAs. The error bars indicate the mean \pm SEM of three independent assays ( $n=12$ mice/group) (one-way ANOVA followed by Tukey's multiple comparison test, $a=0.05$; $\star \star \star, ~ P<0.001$, ns, no significance). (g) Schematic representation of the functional and biological interactions between ENZ resistance and immune evasion accomplished through the FLII/YBX1/PD-L1 regulatory cascade.

\section{Supplementary Files}

This is a list of supplementary files associated with this preprint. Click to download.

- FLllsupplementary.doc 